\title{
Decreasing incidence of ruptured abdominal aortic aneurysm already before start of screening
}

\author{
Sofia Nessvi Otterhag ${ }^{*}$, Anders Gottsäter, Bengt Lindblad and Stefan Acosta
}

\begin{abstract}
Background: The aim of this study was to evaluate whether screening for abdominal aortic aneurysm (AAA) has led to a decrease in ruptured AAA (rAAA) incidence.

Method: The Malmö population was evaluated regarding the incidence of rAAA and elective AAA surgery 4 years before and after start of AAA-screening in 2010. Data from 1971 to 1986 (J Vasc Surg 18:74-80, 1993) and 2000-2004 (J Vasc Surg 44:237-43, 2006), enabled analysis of trends over time.

Results: Analysis of time-periods 1971-1986, 2000-2004, 2006-2010 and 2010-2014 showed an incidence of rAAA of $5.6(4.9-6.3), 10.6(8.9-12.4), 6.1(4.6-7.6)$ and 4.0 (2.9-5.1), respectively. In men aged 60-69 years the incidences were $16.0(10.7-21.3), 45.6(27.7-63.4), 19.3(9.2-35.3)$ and $8.9(2.8-20.6)$, respectively. The incidences of elective AAA surgery in men aged 60-69 years were 22.9 (16.5-29.2), 34.6 (19.1-50.2), 9.7 (1.2-18.5) and 44.2 (27.0-61.6), respectively.

Conclusions: A decrease in incidence of rAAA in men was evident before the implementation of screening. We were yet not able to demonstrate a certain reduction in rAAA incidence after the start of screening.
\end{abstract}

Keywords: Ruptured abdominal aortic aneurysm, Incidence, Population-based study, Autopsy, Screening

\section{Background}

Abdominal aortic aneurysm (AAA) mainly affects men and is a potentially life threatening condition due to the risk for aneurysm rupture (rAAA) [1]. A substantial portion of rAAA patients dies outside hospital [2]. Since AAA is generally asymptomatic before rupture, early detection of the disease is of great clinical importance, and this has been made possible by the introduction of large scale screening programs.

The benefit of screening programs has been supported by four large randomized controlled trials (RCTs) [3-6], in populations from 1988 to 1999 when AAA prevalence rates in 65-year old men ranged between 3.5 and $4.5 \%$. These studies showed a $40 \%$ relative reduction in AAA related death in males [7]. In the MASS study [6], there was a $0.14 \%$ absolute risk reduction in AAA related death $(0.19 \%$ in the screened group and $0.33 \%$ in the control group). However, more recent studies now

\footnotetext{
* Correspondence: sofiaotterhag@gmail.com

Vascular Center, Institution of Clinical Sciences, Lund University, SE-205 02 Malmö, Lund, Sweden
}

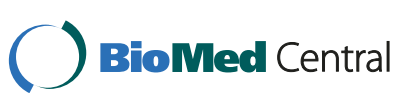

C 2016 Otterhag et al. Open Access This article is distributed under the terms of the Creative Commons Attribution 4.0 International License (http://creativecommons.org/licenses/by/4.0/), which permits unrestricted use, distribution, and reproduction in any medium, provided you give appropriate credit to the original author(s) and the source, provide a link to the Creative Commons license, and indicate if changes were made. The Creative Commons Public Domain Dedication waiver (http://creativecommons.org/publicdomain/zero/1.0/) applies to the data made available in this article, unless otherwise stated. indicate a decreasing prevalence of AAA with rates between approximately $1-2 \%[8-11]$, and the previously demonstrated outcomes of AAA screening should be viewed in this context. Simultaneously, improvements in the surgical management of AAA repair has led to superior outcomes [12], compared to previous studies [3-6].

The aim of the present study was to evaluate the incidence of rAAA and elective AAA surgery, in Malmö, Sweden, before and after introduction of ultrasound AAA screening of men aged 65 years in September 2010. Analysis of trends over time was enabled by results from previously published data from 1971 to 1986 [13] and 2000-2004 [14].

\section{Methods \\ Study population}

This study comprises the population of Malmö 4 years before and 4 years after start of screening for AAA in men aged 65 years, in September 2010. Between 2006 and 2014, the population of Malmö increased from 276,244 to 318,107 (Swedish Central Bureau of Statistics). The 
populations in $2008(286,535)$ inhabitants and 2012 (307,758 inhabitants) were used as the reference populations. The study was approved by the Regional Ethical Review Board in Lund, Sweden.

The evaluation of rAAA incidence alterations over time was possible due to two previous published studies on AAA incidences in Malmö. The population in 1978 (240,000 inhabitants) was used as reference population in the first study [13] (autopsy frequency $85 \%$; Bengtsson et al. 1993), between 1971 and 1986. The second study [14] (autopsy frequency $25 \%$; Acosta et al. 2006) was carried out between 2000 and 2004, with a reference population of 265,481 inhabitants in 2002.

Malmö has one hospital, Skåne University Hospital. During the 8 year study period (September 6, 2010 to September 6, 2014), all surgical procedures and all diagnoses assigned to patients upon discharge or death were classified according to the International Classification of Disease, 10th edition (ICD-10) code and collected in a computerized registry. Data on mortality and domicile was obtained by record linkage with the Swedish Population Registry.

Adherence to the AAA screening program in men aged 65 years, in the population of Malmö, was $80.2 \%$ [15].

\section{Retrieval of rAAA cases}

rAAA was defined as either an extravasation of blood or hematoma outside the AAA -, a diagnosis made with computed tomography $(\mathrm{CT})$, or a hematoma found outside the AAA at open or endovascular aneurysm repair (EVAR), or at autopsy. The ICD-10 code I71.3 was used for identification of patients with rAAA. The Vascular Centre Malmö-Lund is a tertiary referral centre for patients with vascular disease, patients who lived outside Malmö were excluded from this analysis, however.

\section{Retrieval of clinical and forensic autopsy-verified cases of rAAA}

The clinical autopsies were performed according to a standardized detailed protocol at the Department of Clinical Pathology Skåne University Hospital, Lund. Average autopsy rates were $19 \%$ between 2006 and 2010 and $12 \%$ between 2010 and 2014. Findings were coded according to the Systematized Nomenclature of Medicine (SNOMED), as defined by the College of American Pathologists. The topographic code for aorta $(\mathrm{t} 42)$ and the code of diagnosis for aneurysm (m324) or rupture (m1443) were used in a search to identify all individuals with rAAA. A computerized autopsy registry was used to identify patients with rAAA.

Forensic autopsies were performed at the Institution of Forensic Medicine, Lund, upon request from police authorities. A search to identify individuals with rAAA was performed using the code for aortic aneurysm and dissection (441) before 2013 (according to the ICD-9-CM diagnostic codes) and the codes for diagnoses rAAA (I71.3) and ruptured thoracic aneurysm (171.1) after 2013 was used (according to ICD-10-CM diagnostic codes).

\section{Evaluation of elective and acute AAA surgery}

Elective surgery for an AAA was indicated, in the absence of significant comorbidity, if maximal AAA diameter on ultrasound was $>5.0 \mathrm{~cm}(>5.5 \mathrm{~cm}$ in men or $>5.0 \mathrm{~cm}$ in women measured at CT scan). Acute surgery was performed in ruptured AAA. EVAR was the first choice method for both elective and acute AAA repairs between 2006 and 2014.

Patients from Malmö who underwent elective surgery for AAA or acute surgery for rAAA were identified (ICD code I71.4 and I71.3 respectively and codes for open repair [OR] or endovascular aneurysm repair [EVAR]). All patient records were retrieved for verification of the diagnosis.

\section{Statistical methods}

Age- and gender-specific incidence rates were based on the number of cases of rAAA or elective AAA repairs, respectively, and were expressed as number of cases per 100000 person-years. Age- and gender- specific event rates and incidence rates from 2006 to 2010 and 2010 to 2014 were computed using the 2008 and 2012 populations in Malmö, respectively, as reference, and $95 \%$ confidence intervals (CIs) were calculated assuming a Poisson distribution of events, using the exact method for $N<15$ and the normal approximation for larger numbers.

Age in the figures was presented as median (interquartile range $[\mathrm{IQR}]$ ). Differences in proportions were evaluated using the Chi [2] test. Mann-Whitney $U$ tests were used when comparing groups using continuous variables. $P<0.05$ was considered significant.

\section{Results \\ Elective repair of AAA}

Elective EVAR was performed in 74 (97 \%) patients during 2006-2010 and 84 (97\%) Malmö city patients during 2010-2014. Open repair was performed in two and three patients, respectively. The total incidences of elective AAA surgery were $6.6(5.1-7.7)$ and 7.0 (5.5-8.5) (per 100000 person years [95\% CI]), respectively. The ratio of elective versus acute AAA surgery increased from 1.5 (76/50) during 2006-2010 to 2.8 (87/23) during 2010-2014 in the whole population, and from $1.5(58 / 38)$ to $4.4(74 / 17)$ in males and $1.5(18 / 12)$ to $2.2(12 / 6)$ in females, respectively. (Table 1 ).

The median age of patients undergoing elective AAA surgery, 4 years before start of screening was 75 years (IQR 71-81), and decreased to 73 years (68-79) 4 years after start of screening $(p=0.028)$. The proportions of 
Table 1 Epidemiology of abdominal aortic aneurysm (AAA) in Malmö during 1971-1986 [13], 2000-2004 [14], 2006-2010 and $2010-2014$

\begin{tabular}{|c|c|c|c|c|}
\hline Study & $\begin{array}{l}\text { Bengtsson et al. } \\
1971-1986\end{array}$ & $\begin{array}{l}\text { Acosta et al. } \\
2000-2004\end{array}$ & $\begin{array}{l}\text { Before start of screening } \\
2006-2010\end{array}$ & $\begin{array}{l}\text { After start of screening } \\
\text { 2010-2014 }\end{array}$ \\
\hline \multicolumn{5}{|l|}{ Demographic data } \\
\hline Malmo Population & 240000 & 265000 & 285514 & 307207 \\
\hline Percentage $>75$ years & $6 \%$ & $10 \%$ & $9 \%$ & $8 \%$ \\
\hline Ratio F:M in persons $>75$ years & $2: 1$ & 1:9:1 & $3: 2$ & $3: 2$ \\
\hline Autopsy frequency & $85 \%$ & $25 \%$ & $19 \%$ & $12 \%$ \\
\hline \multicolumn{5}{|l|}{ Incidence of $\mathrm{rAAA}^{\mathrm{a}}$} \\
\hline Men & $8.4(7.1-9.7)$ & $18.1(14.8-21.4)$ & $10.5(7.9-13.1)$ & $6.2(4.2-8.2)$ \\
\hline $60-69$ years & $16.0(10.7-21.3)$ & $45.6(27.7-63.4)$ & $19.3(9.2-35.3)$ & $8.9(2.8-20.6)$ \\
\hline 70-79 years & $55.8(42.7-68.9)$ & $117(84.3-149.0)$ & $75.4(45.2-105.6)$ & $44.4(21.9-66.9)$ \\
\hline $80+$ years & $113(75.9-150) 3.0$ & $154(102-206)$ & $99.8(57.2-142.4)$ & $86.4(46.52-126.3)$ \\
\hline Women & $3.0(2.2-3.8)$ & $3.6(2.2-5.1)$ & $2.9(2.3-3.5)$ & $1.9(0.8-3.0)$ \\
\hline 60-69 years & $0.8(0.1-3.6)$ & $6.5(1.8-16.6)$ & $7.2(2.0-18.4)$ & $0(0-6.2)$ \\
\hline 70-79 years & $8.6(4.5-12.7)$ & $11.5(4.6-23.7)$ & $14.1(5.2-30.8)$ & $7.1(1.5-21.7)$ \\
\hline $80+$ years & $43.0(28.3-57.7)$ & $25.1(12.5-44.9)$ & $16.5(6.6-34.0)$ & $22.3(10.1-42.4)$ \\
\hline All & $5.6(4.9-6.3)$ & $10.6(8.9-12.4)$ & $6.1(4.6-7.6)$ & $4.0(2.9-5.1)$ \\
\hline \multicolumn{5}{|l|}{ Surgically treated/all rAAA(\%)cases } \\
\hline Men & $52 / 155(34)$ & $62 / 116(53)$ & $38 / 59(64)$ & $17 / 38(45)$ \\
\hline Women & $9 / 60(15)$ & $5 / 25(20)$ & $12 / 17(71)$ & $6 / 12(50)$ \\
\hline All & $61 / 215(28)$ & $67 / 141(48)$ & $50 / 76(66)$ & $23 / 50(46)$ \\
\hline \multicolumn{5}{|l|}{ Incidence of elective AAA surgery ${ }^{a}$} \\
\hline Men & $5.9(4.7-7.0)$ & $10.1(7.7-12.6)$ & $10.3(7.6-13.0)$ & $12.8(9.9-15.6)$ \\
\hline $60-69$ years & $22.9(16.5-29.2)$ & $34.6(19.1-50.2)$ & $9.7(1.2-18.5)$ & $44.2(27.0-61.6)$ \\
\hline 70-79 years & $33.5(23.4-43.6)$ & $67.7(43.1-92.3)$ & $113.1(76.1-150.1)$ & $88.8(57.0-120.6)$ \\
\hline $80+$ years & $12.5(3.4-32.1)$ & $68.1(33.6-102)$ & $99.8(57.1-142.1)$ & $91.2(50.2-132.2)$ \\
\hline Women & $1.1(0.6-1.6)$ & $4.1(2.6-5.6)$ & $3.1(1.7-4.5)$ & $1.9(0.8-3.0)$ \\
\hline $60-69$ years & $2.8(1.1-5.8)$ & $12.9(5.6-25.5)$ & $5.4(1.1-15.8)$ & $3.3(0-7.8)$ \\
\hline 70-79 years & $5.1(2.4-9.3)$ & $27.9(14.6-41.1)$ & $14.1(5.2-30.1)$ & $16.7(4.4-29.0)$ \\
\hline $80+$ years & $1.3(0.0-7.3)$ & $4.6(0.6-16.5)$ & $18.9(8.0-37.1)$ & $5.0(0-11.8)$ \\
\hline All & $3.4(2.8-4.0)$ & $7.0(5.6-8.4)$ & $6.6(5.1-7.7)$ & $7.0(5.5-8.5)$ \\
\hline \multicolumn{5}{|l|}{ Ratio (N/N) elective/acute surgery } \\
\hline Men & $2.1(108 / 52)$ & $1.0(65 / 62)$ & $1.5(58 / 38)$ & $4.4(75 / 17)$ \\
\hline Women & $2.4(22 / 9)$ & $5.6(28 / 5)$ & $1.5(18 / 12)$ & $2.0(12 / 6)$ \\
\hline All & $2.1(130 / 61)$ & $1.4(93 / 67)$ & $1.5(76 / 50)$ & $2.8(87 / 23)$ \\
\hline
\end{tabular}

Incidence per 100,000 person years $(95 \% \mathrm{Cl})$

males were $73 \%(58 / 76)$ and $86 \%(75 / 87)$, respectively $(p=0.104)$. The incidence of elective AAA surgery in men aged 60-69 years was $22.9(16.5-29.2)$ in 19711986 [13] (Bengtsson et al. 1993), 34.6 (19.1-50.2) in 2000-2004 [14] (Acosta et al. 2006), and decreased significantly to $9.7(1.2-18.5)$ in 2006-2010, and then after start of screening increased significantly to 44.2 (27.061.6) in 2010-2014 (per 100000 person years [95 \% CI]). (Table 1).

\section{Epidemiology of rAAA}

In 2006-2010 and 2010-2014 the diagnosis was established after clinical autopsy in $16(21 \%)$ and 16 (36 \%) patients, respectively, whereas 5 (7\%) and 4 (9\%), respectively, had been referred from the police authorities to the Institution of Forensic Medicine (Table 2). Autopsy rates decreased from $85 \%$ in $1971-1986$, to $25 \%$ in 2000-2004, to $19 \%$ in 2006-2010, and finally to $12 \%$ in 2010-2014 (Table 1, Fig. 2). 
Table 2 Clinical characteristics of rAAA patients in 2000-2004 [14], 2006-2010 and 2010-2014

\begin{tabular}{|c|c|c|c|c|}
\hline \multirow[t]{3}{*}{ Study } & Acosta et al. & Before start of screening & After start of screening & $P$-value \\
\hline & 2000-2004 & 2006-2010 & $2010-2014$ & \\
\hline & N (\%) & N (\%) & N (\%) & \\
\hline \multicolumn{5}{|l|}{ Age } \\
\hline-59 & $3(2)$ & $4(5)$ & 0 & \\
\hline $60-69$ & $29(21)$ & $14(19)$ & $5(10)$ & \\
\hline $70-79$ & $57(41)$ & $30(40)$ & $18(36)$ & \\
\hline $80+$ & $51(36)$ & $28(37)$ & $27(54)$ & 0.168 \\
\hline \multicolumn{5}{|l|}{ Gender } \\
\hline Male & $115(82)$ & $59(78)$ & $38(76)$ & 0.565 \\
\hline \multicolumn{5}{|l|}{ Autopsy } \\
\hline Clinical & $54(40)$ & $16(21)$ & $16(36)$ & 0.104 \\
\hline Forensic & $9(6)$ & $5(7)$ & $4(9)$ & \\
\hline Deaths from rAAA & $103(74)$ & $43(57)$ & $35(70)$ & 0.014 \\
\hline Total & $140(100)$ & $76(100)$ & $50(100)$ & - \\
\hline
\end{tabular}

During the 4 year time periods before and after start of screening in September 2010, 76 versus 50 rAAA patients were identified, respectively. The median age of rAAA patients was 76 (IQR 70-83) years in 2006-2010 and $80(75-85)$ years in 2010-2014 $(p=0.071)$, and the proportions of patients aged 80 years or older were 28 (37\%) and 27 (54\%), respectively. The proportions of men suffering from rAAA during the respective time period, were $59(78 \%)$ and $38(76 \%)$. Before and after start of screening 43 (57\%) out of 76 and 35 (70\%) out of 50 persons suffering from rAAA died $(p=0.014)$ (Table 2). During the 4 year time periods before and after start of screening, in total four men and one man, respectively, aged 65-69 years died from rAAA. In the respective time periods two and 12 65-69-year old men underwent elective AAA surgery, and none died within 30 days of elective surgery. The absolute risk for AAA related mortality 4 years before and after implementation of screening was $1.3 \%(4 / 308$ [AAA related mortality/total mortality of 65-69 year old men]) and $0.3 \%(1 / 300)$, respectively. This corresponds to a relative risk reduction of $75 \%$.

The overall incidences of rAAA were 6.1 (95\% CI 4.6-7.6) per 100000 in 2006-2010 and 4.0 (2.9-4.1) in 2010-2014. An analysis of trend over the time-periods showed a significant increase in the total incidence of rAAA per 100000 person years in men during 19711986 [13] (Bengtsson et al. 1993) and 2000-2004 [14] (Acosta et al. 2006); from 8.4 (7.1-9.7) to 18.1 (14.8-21.4), and then significant decreases in 2006-2010 to 10.5 (7.9-13.1), and and 2010-2014 to $6.2(4.2-8.2)$ (per 100 000 person years [95 \% CI]), respectively (Fig. 1). No corresponding decrease in rAAA incidence was seen among women, with incidences of 3.0 (2.2-3.8), 3.6 (2.2-5.1),
$2.9(2.3-3.5)$, and $1.9(0.8-3.0)$ (per 100000 person years [95\% CI]), respectively. In men aged 60-69 years the incidences of rAAA were 16.0 (10.7-21.3), 45.6 (27.7-63.4), 19.3 (9.2-35.3), and 8,9 (2.8-20.6), respectively (per 100000 person years [95 \% CI]) (Fig. 2, Table 1)

\section{Management of rAAA patients}

Out of the 59 patients in 2006-2010 and 25 in patients 2010-2014 rAAA patients in hospital care for rAAA, 37 (63\%) and 17 (68\%), respectively, were initially managed by vascular or general surgeons and 50 (85\%) and 23 (92\%), respectively, underwent aneurysmal repair. Thus, in-hospital death, without surgery, occurred in nine (15\%) and two (8\%) patients, respectively. In 2006-2010 five out of nine patients were assessed in time and then not considered for surgery: The reasons for non-operative management was high age in three patients, heart failure and severe chronic obstructive disease in the remaining two. During 2010-2014 advanced age was the reason for non-operative management in the one patient assessed in time and not considered for surgery. Five patients between 2006 and 2014 were diagnosed either by clinical examination or CT scan, but died before surgery was possible.

\section{Screening}

During the 4 year period after start of screening in September 2010, a total of 12 (20\%) elective AAA surgeries was performed as a result of aneurysm detection through screening of Malmö city individuals. Two (4 \%) patients who suffered from rAAA had previously been subjected to AAA screening (the maximal transverse aortic aneurysm diameter was 7.5 and $5.0 \mathrm{~cm}$, respectively), one of these patients underwent acute surgery for rAAA. Both patients 


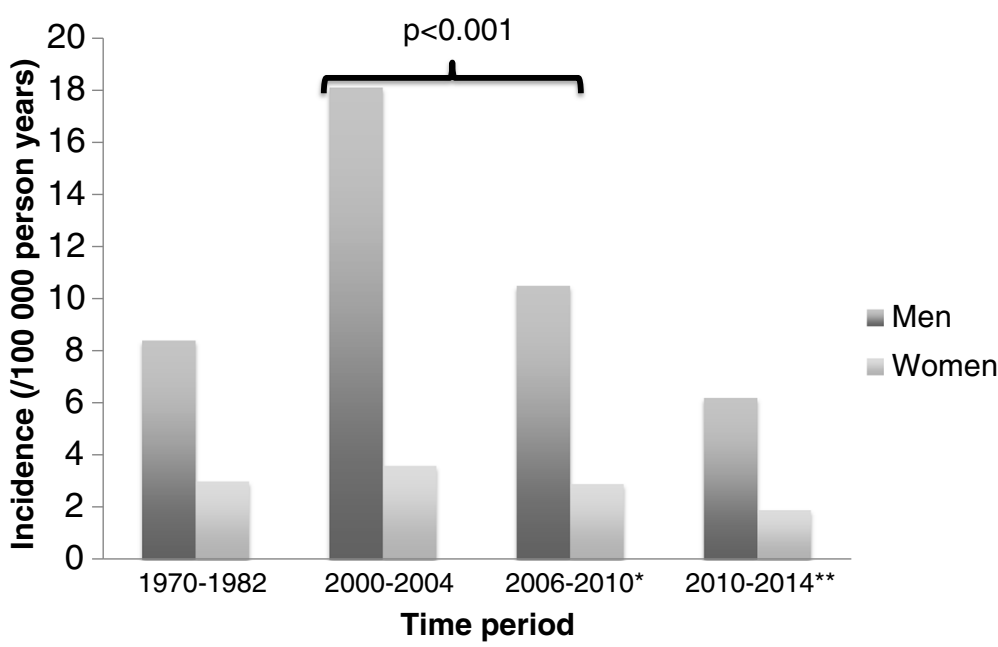

Fig. 1 Total population incidence of ruptured abdominal aortic aneurysm in Malmö between 1970 and 2014. *September 2006 - September 2010. Before start of screening. ${ }^{* *}$ September 2010 - September 2014. After start of screening

were considered for elective repair, one patient was on the waiting list for surgery and the other was denied repair because of co-morbidities.

\section{Discussion}

The present study is unique, as it is based on the Malmö city population during four decades, and takes the findings in both clinical and forensic post mortem examinations as well as the current autopsy rates into account. Aneurysm rupture is fatal in $74-90 \%$ of cases $[2,14,16$, 17], and about $32 \%$ die outside hospital [2]. Accurate information on prehospital mortality and non-intervention rates in rAAA has been scarce in recent studies [2]. The high average autopsy frequency in the Malmö population between 1971 and 1986 [13] allowed a thorough approximation of the incidence of rAAA. With the decreasing autopsy frequency in recent decades, the diagnostic insecurity rises [18] and generates increased risk of bias towards an underestimation of the true incidence of rAAA [19-21].

In comparison with the other time frames, the peak of total incidence rates of rAAA between 2000 and 2004, might partly be explained by the higher proportion of elderly. This study demonstrates a reduction in the overall incidence of rAAA in men during the last decade, even before start of AAA screening in the autumn of 2010. This finding is in concordance with previous studies, showing both decreasing rAAA incidence and AAA prevalence during the 21 st century $[2,8,22]$. This might be explained by improved control of risk factor for AAA development, including improved antihypertensive and lipid lowering treatment. Most importantly, smoking causes $75 \%$ of AAA cases in the population $[8,23]$ and is the single most important modifiable risk-factor for AAA

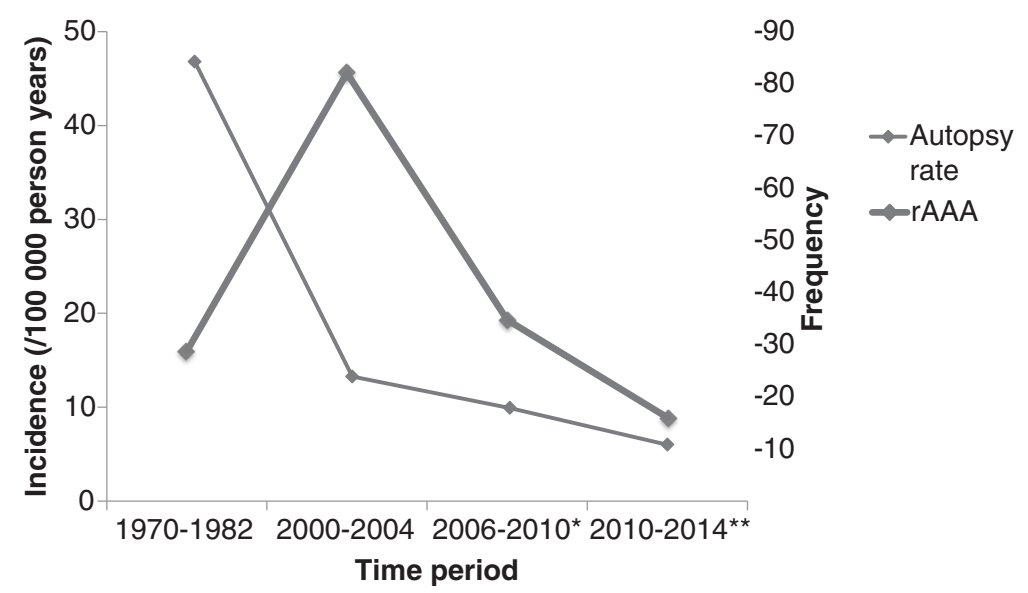

Fig. 2 Autopsy rate and incidence of ruptured abdominal aortic aneurysm (rAAA) in men, between 60 and 69 years of age, in Malmö. *September 2006 - September 2010. Before start of screening. ** September 2010 - September 2014. After start of screening 
development. It has been shown that active smoking increases the risk of aneurysm rupture $[16,24,25]$ and that the continuous trend of decreasing smoking prevalence $[8,26,27]$ amongst age-groups at risk of aneurysm rupture is an essential contributing factor to the decreasing incidence of rAAA. In Sweden, daily smoking among 65-74 year-old men has decreased from $32 \%$ in 1980 to $12.5 \%$ in 2013 [28].

In the age group of men subjected to screening, we found no significant reduction in the incidence of rAAA. Encouraging results from four large randomized screening trials $[3,4,6,29]$, randomizing male population in 1980-1999 with AAA prevalence rates of 4-7.5 \%, have led to the implementation of national screening-programs. With the decreasing AAA prevalence rates, the benefit of AAA screening of all men has been questioned, however. The U.S Preventive Services Task Force recommends screening for all 65-year old men who have ever smoked [30]. Another question that needs further investigation is the appropriate age for screening. A study by Svensjö et al. [8] found only a $1.7 \%$ prevalence of screening-detected AAA in 65-year-old men in Sweden. Additionally, with an increased life expectancy [28], patients have a longer lifespan after screening, during which they are at risk for late AAA development. Studies have also indicated a shift in AAA disease to affect older patients [8,31], meaning that patients with normal aortic diameter at screening at age 65 still are at risk of developing AAA [32]. Despite the relatively short time of follow-up in the present study, interestingly, two patients who underwent screening still suffered from aneurysm rupture. As rAAA is a condition associated with a high mortality and as AAA is generally asymptomatic before rupture, the disease seems well suited for screening. Though, with the increasing use of radiologic imaging in medical practice, incidental AAA findings are common [33] and might impact the costeffectiveness of screening in an era of decreasing AAA prevalence.

In the present study, we found a high rAAA mortality rate during 2010-2014. This might be explained by the fact that over $50 \%$ of rAAA patients were aged 80 years or older, with age-related comorbidities. We also found an increased ratio of elective AAA treatment and a lower age of elective AAA treatment in males following the start of AAA screening in males. Hence, 65-year old males with AAA were likely identified by screening and electively treated, whereas in the non-screened population above 80 years aneurysms might remain unidentified until rupture. As these findings were not seen in women, they might be a consequence of screening, even if no significant reduction in incidence of rAAA in men could be demonstrated. The total incidence of elective AAA repair has decreased significantly during the past 14 years, explained by the falling AAA prevalence during the last decade $[8,9,26]$.
The study limitations includes the potential risk for type two-error in not detecting a significant reduction of rAAA, due to the relatively short time of follow-up after start of AAA screening and the low incidence of rAAA. Furthermore, data from previous study periods should be interpreted with caution, and the considerable reduction in autopsy rate especially among the elderly [34] might have biased the assessment of rAAA incidence towards an underestimation.

\section{Conclusions}

This study confirms the reduction in the incidence of rAAA in men since the beginning of the 21st century $[2,8,20,22]$. This might be explained by an improved control of cardiovascular risk factors and in particular decreasing smoking rates. We were not able to demonstrate a reduction in rAAA incidence 4 years after start of screening, however, and it is important to continue assessing the effects of screening in the future.

\section{Competing interests}

The authors declare that they have no competing interests.

\section{Authors'contributions}

SNO performed the statistical analysis, collected data and drafted the manuscript. AG participated in the design and coordination of the study and helped to draft the manuscript. BL participated in the design of the study and helped to draft the manuscript. SA conceived of the study, was part of design and coordination and participated in drafting the manuscript. All authors read and approved the final manuscript.

\section{Acknowledgements}

This research received no specific grand from any funding agency in the public, commercial, or not-for-profit sectors.

Received: 23 July 2015 Accepted: 8 February 2016

Published online: 17 February 2016

\section{References}

1. Sakalihasan N, Limet R, Defawe OD. Abdominal aortic aneurysm. Lancet. 2005;365:1577-89.

2. Reimerink JJ, van der Laan MJ, Koelemay MJ, Balm R, Legemate DA. Systematic review and meta-analysis of population-based mortality from ruptured abdominal aortic aneurysm. Br J Surg. 2013;100:1405-13.

3. Scott R, Wilson N, Ashton H, Kay D. Influence of screening on the incidence of ruptured abdominal aortic aneurysm: 5-year results of a randomized controlled study. Br J Surg. 1995;82:1066-70.

4. Norman P, Jamrozik K, Lawrence-Brown M, Le M, Spencer C, Tuohy R, et al. Population based randomised controlled trial on impact of screening on mortality from abdominal aortic aneurysm. BMJ. 2004;329:1259.

5. Lindholt J, Sørensen J, Søgaard R, Henneberg E. Long-term benefit and cost-effectiveness analysis of screening for abdominal aortic aneurysms from a randomized controlled trial. Br J Surg. 2010;97:826-34.

6. Ashton HA, Buxton MJ, Day NE, Kim LG, Marteau TM, Scott RA, et al. The multicentre aneurysm screening study (MASS) into the effect of abdominal aortic aneurysm screening on mortality in men: a randomised controlled trial. Lancet. 2002;360(9345):1531-9.

7. Cosford P, Leng G. Screening for abdominal aortic aneurysm. Cochrane Database Syst Rev. 2007;2:CD002945.

8. Svensjö S, Björck M, Gurtelschmid M, Djavani Gidlund K, Hellberg A, Wanhainen A. Low prevalence of abdominal aortic aneurysm among 65 -year-old Swedish men indicates a change in the epidemiology of the disease. Circulation. 2011;124:1118-23.

9. Norman PE, Spilsbury K Semmens JB. Falling rates of hospi- talization and mortality from abdominal aortic aneurysms in Australia. J Vasc Surg. 2011;53:274-77. 
10. Anjum A, von Allmen R, Greenhalgh R, Powell JT. Explaining the decrease in mortality from abdominal aortic aneurysm rupture. Br J Surg. 2012;99:637-45.

11. Darwood R, Earnshaw J, Turton G, Shaw E, Whyman M, Poskitt K, et al. Twenty-year review of abdominal aortic aneurysm screening in men in the county of Gloucestershire, United Kingdom. J Vasc Surg. 2012;56:8-13.

12. Mani K, Björck M, Wanhainen A. Changes in the management of infrarenal abdominal aortic aneurysm disease in Sweden. Br J Surg. 2013;100:638-44.

13. Bengtsson $H$, Bergqvist $D$. Ruptured abdominal aortic aneurysm-a population-based study. J Vasc Surg. 1993;18:74-80.

14. Acosta S, Ögren M, Bengtsson H, Bergqvist D, Lindblad B, Zdanowski Z. Increasing incidence of ruptured abdominal aortic aneurysm: A populationbased study. J Vasc Surg. 2006;44:237-43.

15. Zarrouk M, Holst J, Malina M, Lindblad B, Wann-Hansson C, Rosvall M, et al. The importance of socioeconomic factors for compliance and outcome at screening for abdominal aortic aneurysm in 65-year-old men. J Vasc Surg. 2013:58(1):50-5

16. Brown $L C$, Powell JT. Risk factors for aneurysm rupture in patients kept under ultrasound surveillance. UK Small Aneurysm Trial Participants. Ann Surg. 1999;230:289-96.

17. Ballard DJ, Filardo G, Fowkes FG, Powell JT. Surgery for small asymptomatic abdominal aortic aneurysms. Cochrane Database Syst Rev. 2000;2:CD001835.

18. Royal College of Pathologists of Australasia Autopsy Working Party. The decline of the hospital autopsy: a safety and quality issue for healthcare in Australia. Med J Aust. 2004;180(6):281-85.

19. Xiao J, Krueger GR, Buja LM, Covinsky M. The impact of declining clinical autopsy: need for revised healthcare policy. Am J Med Sci. 2009:337:41-6.

20. Shojania KG, Burton EC. The vanishing nonforensic autopsy. N Engl J Med. 2008:358:873-75.

21. Nashelsky MB, Lawrence CH. Accuracy of cause of death determination without forensic autopsy examination. Am J Forensic Med Pathol. 2003;24:313-19.

22. Sandiford P, Mosquera D, Bramley D. Trends in incidence and mortality from abdominal aortic aneurysm in New Zealand. Br J Surg. 2011;98:645-51.

23. Lederle FA, Johnson GR, Wilson SE, Chute EP, Hye RJ, Makaroun MS, et al. The aneurysm detection and management study screening program: validation cohort and final results. Aneurysm detection and management veterans affairs coop- erative study investigators. Arch Intern Med. 2000:160:1425-30

24. Jahangir E, Lipworth L, Edwards TL, Kabagambe EK, Mumma MT, et al. Smoking, sex, risk factors and abdominal aortic aneurysms: a prospective study of 18782 persons aged above 65 years in the southern community cohort study. J Epidemiol Community Health. 2015;69:481-88.

25. Sweeting MJ, Thompson SG, Brown LC, Powell JT, on behalf of the RESCAN collaborators. Meta-analysis of individual patient data to examine factors affecting growth and rupture of small abdominal aortic aneurysms. Br J Surg. 2012;99:655-65.

26. Anjum A, Powell JT. Is the incidence of abdominal aortic aneurysm declining in the 21st century? Mortality and hospital admissions for England \& Wales and Scotland. Eur J Vasc Endovasc Surg. 2012:43:161-66.

27. Svensjö S, Björck M, Wanhainen A. Update on screening for abdominal aortic aneurysm: a topical review. Eur J Vasc Endovasc Surg. 2014:48:659-67.

28. SCB. Statistiska Centralbyrån [Statistics Sweden]. www.scb.se. Accessed April 13, 2015.

29. Lindholt JS, Juul S, Fasting H, Henneberg EW. Hospital costs and benefits of screening for abdominal aortic aneurysms. Results from a randomised population screening trial. Eur J Vasc Endovasc Surg. 2002;23:55-60.

30. Guirguis-Blake JM, Beil TL, Senger CA, Whitlock EP. Ultrasonography screening for abdominal aortic aneurysms: a systematic evidence review for the U.S. Preventive services task force. Ann Int Med. 2014;1:1-9.

31. Choke E, Vijaynagar B, Thompson J, Nasim A, Bown MJ, Sayers RD. Changing epidemiology of abdominal aortic aneurysms in England and wales older and more benign? Circulation. 2012;125:1617-25.

32. Hafez $\mathrm{H}$, Druce PS, Ashton HA. Abdominal aortic aneurysm development in Men following a "normal" aortic ultrasound scan. Eur J Vasc Endovasc Surg. 2008:36:553-58

33. van Walraven C, Wong J, Morant K, Jennings A, Jetty P, Forster AJ. Incidence, follow-up, and outcomes of incidental abdominal aortic aneurysms. J Vasc Surg. 2010;52:282-89.

34. Causes of Death. Report from the National Board of Health and Social Welfare. Published 2013, Table 11, pages 45 and $252-253$ 